Rodrigo Dresdner Cid, PhD (*)

Jorge Oscar Folino, PhD (**)

Cita:

Rodrigo Dresdner; Jorge Folino. Trastornos mentales y responsabilidad criminal en

incendiarios. Revista Española de Medicina Legal. Volume 43, Issue 2, April-June 2017, Pages

$58-63$

December 2016, DOI: 10.1016/j.reml.2016.10.002

*Psiquiatra Forense y Médico Coordinador de la Unidad de Psiquiatría Adultos; Servicio Médico Legal; Santiago; Chile.

**Psiquiatra Forense y Profesor de Psiquiatría; Facultad de Ciencias Médicas; Universidad Nacional de La Plata, Argentina. 
Correspondencia Rodrigo F. Dresdner Cid, Email: rf_dresdner @yahoo.com.ar Fono: 56222783528 - 223330513. 


\section{TRASTORNOS MENTALES Y RESPONSABILIDAD CRIMINAL EN}

INCENDIARIOS. Mental disorders and criminal responsibility in arsonists.

\section{Resumen}

Introducción: Los objetivos del estudio son describir a acusados del delito de incendio en las dimensiones demográfica, psiquiátrica y criminológica y explorar factores asociados a la opinión pericial de inimputabilidad. Material y métodos: Se describe la serie de casos acusados de incendio y evaluados pericialmente $(N=197)$ en la Unidad de Psiquiatría Adultos del Servicio Médico Legal de Chile, durante el periodo 1999 - 2012 y con diseño de casos y controles se exploran factores asociados con la opinión de inimputabilidad. Resultados: La serie de casos tuvo alta prevalencia de problemas sociales, familiares y de trastornos mentales. En el $52,3 \%$ se alcanzó conclusión pericial compatible con la imputabilidad, en el $24,4 \%$ con la inimputabilidad y en el $23,3 \%$ con la imputabilidad disminuida. Conclusiones: Las condiciones de inimputabilidad e imputabilidad disminuidas, agrupadas como "opinión pericial de imputabilidad comprometida" se asociaron significativamente a la verificación de conducta bizarra, motivación patológica, motivación afectiva, autolesiones y actitud colaboradora del acusado.

Palabras claves: incendio; incendiario; evaluación psiquiátrica; imputabilidad; trastorno mental.

\section{Abstract:}

Introduction: The purpose of the study is to describe arsonists in their demographic, psychiatric and criminological dimensions and to explore factors associated with the expert opinion of insanity. Material and methods: a series of cases accused of arson and assessed in the adult Psychiatric Unit of the Legal Medical Service of Chile, during the period $1999-2012(N=197)$ is described. With a cases and controls design, the factors associated with the opinion of insanity are explored. Results: The series of cases had a high prevalence of mental disorders, social and family problems. Expert opinion compatible with the condition of criminal responsibility was achieved in $52.3 \%$ of the cases; and in $24.4 \%$ it was compatible with insanity and in $23.3 \%$ with diminished criminal responsibility. Conclusions: The conditions of insanity and diminished criminal responsibility, grouped together as "expert opinion of altered criminal responsibility" were significantly associated with bizarre behavior, 
pathological motivation, emotional motivation, self-harm and cooperative attitude of the accused.

Keywords: arson; arsonist; psychiatric assessment; criminal responsibility; mental disorder. 


\section{Introducción.}

Previos estudios ponen en evidencia que quienes cometen incendios tienen heterogéneas motivaciones ${ }^{i}$ ii $\mathrm{y}$ alta prevalencia de diversos trastornos mentales, principalmente trastornos por consumo de sustancias, psicosis, trastornos de personalidad y retraso mental iii iv $v$ vi vii viii ix $x$ xi $x$ xi $x$ xii xiv $x v$. El diagnóstico clínico de "piromanía" ha sido cuestionado en cuanto a su validez teórica y utilidad para la investigación forense ya no se corresponde con la mayoría de los incendiarios xvi xvii. También se reporta que en esta población los trastornos de personalidad constituyen uno de los diagnósticos psiquiátricos más frecuentes xviii xix $x x$. Investigaciones sobre psicopatía en población incendiaria no agregan particularidades en este tipo de sujetos distintos de las descritas de la psicopatía en general ${ }^{\times x i}$ xxii.

Otros autores reportan que es frecuente encontrar problemas socioeconómicos $y$ dificultades relacionales xxiii xxiv $y$ relacionan la conducta incendiaria con factores psicológicos, emocionales, anímicos, sexuales, descontrol de impulsos, consumo de sustancias, intenciones suicidas e intereses económicos xxv xxvi xxvii.

Mientras que Ganon (2010) señala que las mujeres constituyen un grupo poco estudiado, otros autores reportan un aumento absoluto y relativo de hasta un $38 \%$ del total en poblaciones de incendiarios xxviii xxix. Algunos estudios en mujeres incendiarias informan que la frecuencia de los diagnósticos psiquiátricos fue mayor que entre los varones, llegando a prevalencias del 42 al $92 \%$, y también que las mujeres tuvieron alta frecuencia de comportamientos

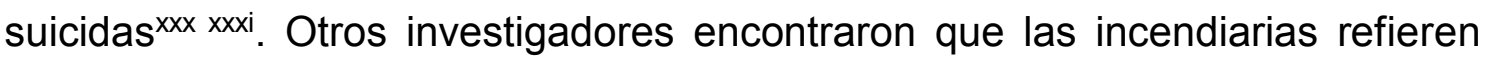
abundantes antecedentes infantiles de abusos y deprivación y de manera similar a como lo hacen las mujeres delincuentes no incendiarias xxxii xxxiii xxxivxxxv. Bourget (1989), por su parte, observó que las incendiarias comparadas con mujeres no delincuentes tenían mayor probabilidad de ser solteras 0 separadas.

De estos aportes ninguno proviene de estudios latinoamericanos y de Chile se tiene un único estudio descriptivo (Koppmann et al, 1998). Dicho de otro modo, poco sabemos sobre cómo son los incendiarios locales, qué trastornos tienen, 
qué motivaciones los lleva a cometer incendios y qué similitudes y diferencias tienen con los incendiarios de otros países, a pesar que esa información resultaría relevante como insumo para políticas públicas y planes de prevención primaria y secundaria.

Con el objetivo de contribuir a completar tal información faltante, en este artículo se describe la serie de casos de incendiarios estudiada en el Servicio Médico Legal de Chile, durante 13 años, atendiendo a sus dimensiones demográficas, clínicas y criminológicas. Asimismo, se explora la relación entre esas variables y la opinión pericial compatible con la inimputabilidad.

\section{Material y métodos.}

Se utilizó un diseño cuantitativo conformado por una sección descriptiva de serie de sujetos acusados de incendio y otra sección analítica con un diseño de casos y controles. En la segunda sección, los casos fueron definidos por la variable dependiente "opinión pericial compatible con la inimputabilidad". Dado que el Código Penal de Chile xxxi estipula, en sus artículos 10 y 11, dos condiciones de compromiso de la responsabilidad penal, a saber, la Inimputabilidad y la Imputabilidad disminuida, para el análisis final, ambas variantes del compromiso de la imputabilidad fueron subsumidas en la variable dependiente "opinión pericial compatible con la imputabilidad comprometida". La hipótesis alternativa planteada se formuló de la siguiente manera: factores demográficos, psiquiátricos y criminológicos se asocian diferencialmente con la condición de ser incendiario con "opinión pericial compatible con la imputabilidad comprometida" y la de serlo pero con "opinión pericial compatible con imputabilidad".

La información fue obtenida de 197 informes psiquiátricos que correspondieron a la totalidad de personas imputadas por el delito de incendio y examinadas en la Unidad de Psiquiatría Adultos del Servicio Médico Legal de Chile durante el periodo 1999 - 2012xxvvii. Si bien la información de los casos juzgados es pública, el procedimiento se realizó con la autorización de la dirección del Servicio Médico Legal y con el reparo de mantener los casos sin nominación. 
Para los diagnósticos se utilizó el DSM IV TR (APA, 2003) y los diagnósticos nosológicos afines fueron agregados en tipos sindromáticos. Las motivaciones incendiarias se categorizaron y se definieron operativamente como se describe a continuación: Afectiva -asociada a sentimientos, estados pasionales y emocionales no patológicos-; psicopatológica -explicable a partir de cualquier trastorno mental del Eje I, incluida la piromanía-; imprudente -derivada de conductas no intencionadas y por descuidos-; criminal -relacionada con intención delictual calculada y planificada, ya sea el incendio parte del objetivo principal o solamente un medio para la comisión de otro delito-; ideológica/política/religiosa -justificada por determinada visión del mundo sostenida por alguna organización a la que el ejecutor pertenece-. Si bien la categorización de estas conductas es difícil e imperfecta por la posible combinación de motivaciones, desde el punto de vista de la salud mental y las ciencias del comportamiento es útil la aproximación.

Los datos obtenidos se ordenaron en tablas de distribución de frecuencia y asociación de variables y para su análisis se utilizó el paquete estadístico SPSS 15.0. Se conformaron los modelos Chi Cuadrado y Regresión Logística y expusieron los resultados obtenidos de la exploración multivariada.

\section{Resultados.}

El promedio de edad de la serie fue 39 años $(D E=13)$. La proporción entre varones y mujeres fue de 4 a 1 y entre ambos sexos no se encontraron diferencias significativas en las variables sociodemográficas, psiquiátricas ni en la imputabilidad. Al tiempo del incendio el $64,5 \%$ se encontraba sin cónyuge o pareja y el $85 \%$ carecía de capacitación laboral o profesional. El 1,5\% tenía el antecedente penal de incendio.

La mayoría presentó algún tipo de diagnóstico psiquiátrico en el Eje I del DSM IV-TR y los trastornos relacionados con consumo de sustancia, siendo el diagnóstico sindromático más frecuente se desglosó en 32,3\% intoxicación por alcohol; $4,8 \%$ otros por abuso de alcohol; $4 \%$ abuso de otras sustancias. La frecuencia del diagnóstico de piromanía fue muy menor. 
La presencia de trastornos del Eje II del DSM IV-TR también fue verificada en una amplia proporción. Todos los trastornos de personalidad fueron agrupados excepto el de tipo evitativo dado que no se pesquisó ningún caso. Se dejó aparte a la psicopatía para cuyo diagnóstico el perito evaluador había utilizado los criterios de Hare (2003) ${ }^{\mathrm{xxxviii}}$ mostrando una baja frecuencia.

\section{AQUÍ VA TABLA N²}

Prácticamente la mitad de la población estudiada arrojó algún grado de compromiso en la imputabilidad y, por ende, en la responsabilidad criminal.

\section{AQUÍ VA TABLA Nº 3}

Como motivación incendiaria destacaron las de tipo afectiva y psicopatológica (Gráfico 1).

\section{AQUÍ VA GRÁFICO Nº 1}

En la mayoría de los casos, el incendiario actuó sin acompañante e incendió su propia casa, resultando infrecuente el compromiso de una segunda o ajena vivienda. La intencionalidad incendiaria estuvo presente en la mayoría de los casos, si bien la proporción de casos con planificación previa fue mucho menor. Posteriormente al incendio lo más frecuente fue la no colaboración con la justicia mientras que el restante se repartió entre comportamientos bizarros o de colaboración. Las consecuencias del incendio desde el punto de vista penal fueron daños a la propiedad seguidos muy lejos por lesiones y fallecimientos. ${ }^{1}$

Se encontró que cinco variables independientes de tipo criminológico y una de tipo jurídico-penal mostraron asociación significativa con la condición de tener “opinión pericial compatible con la imputabilidad comprometida” (Tabla № 4).

\section{AQUÍ VA TABLA Nº 4}

\section{Discusión.}

El objetivo de este estudio fue describir una serie de casos judiciales en los que las personas fueron acusadas de haber cometido incendio y evaluadas

\footnotetext{
${ }^{1}$ La suma de porcentajes es mayor a 100 porque hubo casos en que el incendio ocasionó más de un perjuicio
} 
psiquiátricamente durante el proceso judicial en Chile. Si bien los hallazgos orientan a considerar que esta población de incendiarios tiene características heterogéneas que impiden establecer un perfil único, también permiten discutir algunas regularidades relevantes.

Entre los hallazgos se destaca la alta prevalencia de problemas sociales, familiares y de trastornos mentales, concordantemente con informes de otras latitudes (Enayatti et al, 2008). Específicamente, es muy llamativa la alta prevalencia de problemas por consumo de alcohol. El alcohol, además de tener la potencialidad de actuar como elemento facilitador de conductas violentas, es un indicador de diversas vulnerabilidades pues su consumo suele asociarse a situaciones de conflicto, estrés y desesperanza. Si bien la condición de intoxicación al momento del hecho no tiene una traducción directa sobre la inimputabilidad, que en el presente estudio haya sido diagnosticada en un tercio de los incendiarios y que sea indicadora de situaciones extremas y de eventual desinhibición, orientan a considerarla un factor relevante en la comprensión del fenómeno. De esta manera, se constituye en un elemento que no debería descuidarse a la hora del estudio pericial.

Esta población de incendiarios dista de tener la predominante caracterización antisocial que suele encontrarse en población penitenciaria latinoamericana xxxix xl xil. Además de verificarse una mucho menor proporción de psicopatía que en la población penitenciaria general, llama la atención que en menos de un décimo de la serie los peritos alcancen a determinar la motivación criminal, esto es, relacionada con intención delictual calculada y planificada, ya sea que el incendio sea parte del objetivo principal o solamente un medio para la comisión de otro delito. Teniendo eso en consideración, sería esperable que se hubiera encontrado una preponderante actitud de colaboración con la justicia. Sin embargo, el hallazgo se aleja de lo esperable pues casi la mitad de los incendiarios no colaboraron. La interpretación del hallazgo es que, por una parte, aún en sujetos con rasgos antisociales de personalidad es posible verificar que el comportamiento incendiario se puede asociar a motivaciones afectivas o psicopatológicas y no ser, necesariamente, del tipo delictual. La alternativa puede ser de valor para la investigación policial de este tipo de delito, pues orienta a una búsqueda multidireccional del móvil y posibilita 
comprender mejor la dinámica criminal. Por otra parte, el hallazgo pone de manifiesto que comportamientos evasivos de la responsabilidad como huir, ocultarse o elaborar una coartada se verifican en diversos tipos de incendiarios, aún en aquellos que no tienen trastorno antisocial de personalidad y que, más aún, pueden padecer patologías que comprometen la imputabilidad.

Uno de los principales aportes que hace el estudio es la caracterización de la amplia mayoría de los actos incendiarios como autodestructivos. Los perjuicios del incendio se concentraron notablemente en la propiedad de los mismos incendiarios, lo que conlleva perjuicio económico y consecuencias negativas para la salud y el bienestar propio y de los cohabitantes. La muy frecuente intencionalidad incendiaria detectada no se acompañó de planificación y, además, respondió a motivaciones afectivas, psicopatológicas y por imprudencia. Todos esos hitos de la secuencia comportamental orientan a sostener una dinámica predominantemente reactiva. Consistentemente, la verificación de piromanía fue la excepción. Los hallazgos en general permiten configurar un perfil de los incendiarios del centro de Chile como distante de aquel que puede leerse en crónicas rojas de los medios de comunicación plenos de psicopatía o piromanía que comete incendios de manera serial. Por el contrario, el perfil más representativo es el de una persona con diversos factores de vulnerabilidad, incluyendo el factor psicopatológico, situada en un contexto conflictivo de tipo personal y/o con su entorno cercano.

Otro hallazgo remarcable es la alta proporción de sujetos que carecieron de plena conciencia o discernimiento, o de voluntad al provocar el incendio y de quienes los estudios periciales alcanzaron conclusiones compatibles con compromiso de la imputabilidad. El hallazgo contrasta notablemente con previos estudios latinoamericanos que informaron proporciones de inimputables entre los procesados en general del $0,2 \%$ xlii y en abusadores sexuales del $1 \%$ xliii ( $R$ Dresdner et al, 2005), y solamente resulta cercano a lo informado para homicidas $^{x l i v}$ (JO Folino, M Apezteguía, 2000). De acuerdo a los resultados de este estudio, entre quienes son procesados por haber cometido incendio, es alta la probabilidad de presentar trastorno mental y tener algún grado de afectación del grado de discernimiento y de la voluntariedad que la justicia requiere para poder imputar el delito. Ambos hallazgos aportan información 
valiosa para el servicio de justicia y para el de salud mental. Mientras en el primer ámbito las vulnerabilidades y su impacto en la imputabilidad deberán ser foco de cuidadoso escrutinio a la hora de juzgar al acusado de incendio, en el ámbito de la salud mental, deberá ser cuidadosamente contemplado el riesgo de qué personas con psicopatología y/o cursando situaciones conflictivas cometan incendio. En cuanto a la actividad pericial específica de evaluar y asesorar sobre el estado psíquico al momento, los hallazgos del presente estudio en el modelo de regresión logística resultan también informativos. Al respecto cabe destacar que si bien la asociación entre conducta bizarra y motivación patológica con la opinión de imputabilidad comprometida era esperable, la significativa asociación con motivación afectiva, autolesiones y actitud colaboradora se constituye en un aporte empírico.

El estudio tiene la limitación de que la serie de casos está restringida al centro del país y no es representativa de otras regiones, como la VIII y la IX, donde los incendios intencionales son un problema frecuente. Queda abierta una importante heurística en la medida en que los hallazgos presentes puedan compararse con nuevos estudios en el sur del país. 
Tabla N 1. Distribución de grupos diagnósticos psiquiátricos

\begin{tabular}{|l|l|l|}
\hline Grupo diagnóstico & $\mathrm{N}$ & $\%$ \\
\hline Trastornos por consumo de sustancias & 81 & 41,1 \\
\hline Trastornos psicóticos & 36 & 18,3 \\
\hline Trastornos del ánimo & 16 & 8,1 \\
\hline Demencia, delirium y otros deterioros cognitivos & 15 & 7,6 \\
\hline Otros diagnósticos & 21 & 10,7 \\
\hline Sin diagnóstico & 28 & 14,2 \\
\hline Total & 197 & 100 \\
\hline
\end{tabular}

Nota. Categorías diagnósticas agrupadas del Eje I DSM TR

Tabla 2. Distribución de grupos diagnósticos psiquiátricos

\begin{tabular}{|l|c|c|}
\hline Grupo diagnostico & $\mathrm{n}$ & $\%$ \\
\hline Sin diagnóstico & 72 & 36,6 \\
\hline $\begin{array}{l}\text { Trastorno de } \\
\text { personalidad }\end{array}$ & 104 & 52,8 \\
\hline Retardo mental & 17 & 8,6 \\
\hline Psicopatía & 3 & 1,5 \\
\hline Inteligencia Limítrofe & 1 & 0,5 \\
\hline Total & 197 & 100 \\
\hline
\end{tabular}

Nota. Categorías diagnósticas agrupadas del Eje II DSM TR y especificación de psicopatía 
Tabla N 3. Diagnósticos psiquiátricos del Eje I DSM IV TR y opinión pericial

\begin{tabular}{|l|r|r|r|r|}
\hline Grupo diagnóstico & & \multicolumn{3}{|c|}{ Condición de imputabilidad } \\
\hline & & Imputable & Inimputable & $\begin{array}{l}\text { Imputabilid } \\
\text { ad } \\
\text { disminuida }\end{array}$ \\
\hline Simulación de enfermedad mental & $\%$ & 100,0 & 0.0 & 0,0 \\
\hline Sin diagnóstico & $\%$ & 96,4 & 0,0 & 3,6 \\
\hline Otros diagnósticos no especificados & $\%$ & 0,0 & 0,0 & 100,0 \\
\hline $\begin{array}{l}\text { Demencias/delirium/otros trastornos } \\
\text { cognitivos }\end{array}$ & $\%$ & 20,0 & 53,3 & 26,7 \\
\hline Piromanía & $\%$ & 100,0 & 0,0 & 0,0 \\
\hline Trastorno disociativo & $\%$ & 0,0 & 8,3 & 91,7 \\
\hline Trastorno adaptativo & $\%$ & 60,0 & 0,0 & 40,0 \\
\hline Trastorno afectivo mayor sin psicosis & $\%$ & 0,0 & 20,0 & 80,0 \\
\hline Trastorno psicótico & $\%$ & 2,1 & 80,9 & 17,0 \\
\hline Intoxicación por sustancias & $\%$ & 79,1 & 0,0 & 20,9 \\
\hline Abuso de sustancias & $\%$ & 100,0 & 0,0 & 0,0 \\
\hline Dependencia de sustancias & $\%$ & 75,0 & 0,0 & 25,0 \\
\hline Total & $\%$ & 52,3 & 24,4 & 23,4 \\
\hline
\end{tabular}




\section{Gráfico 1. Motivaciones incendiarias}

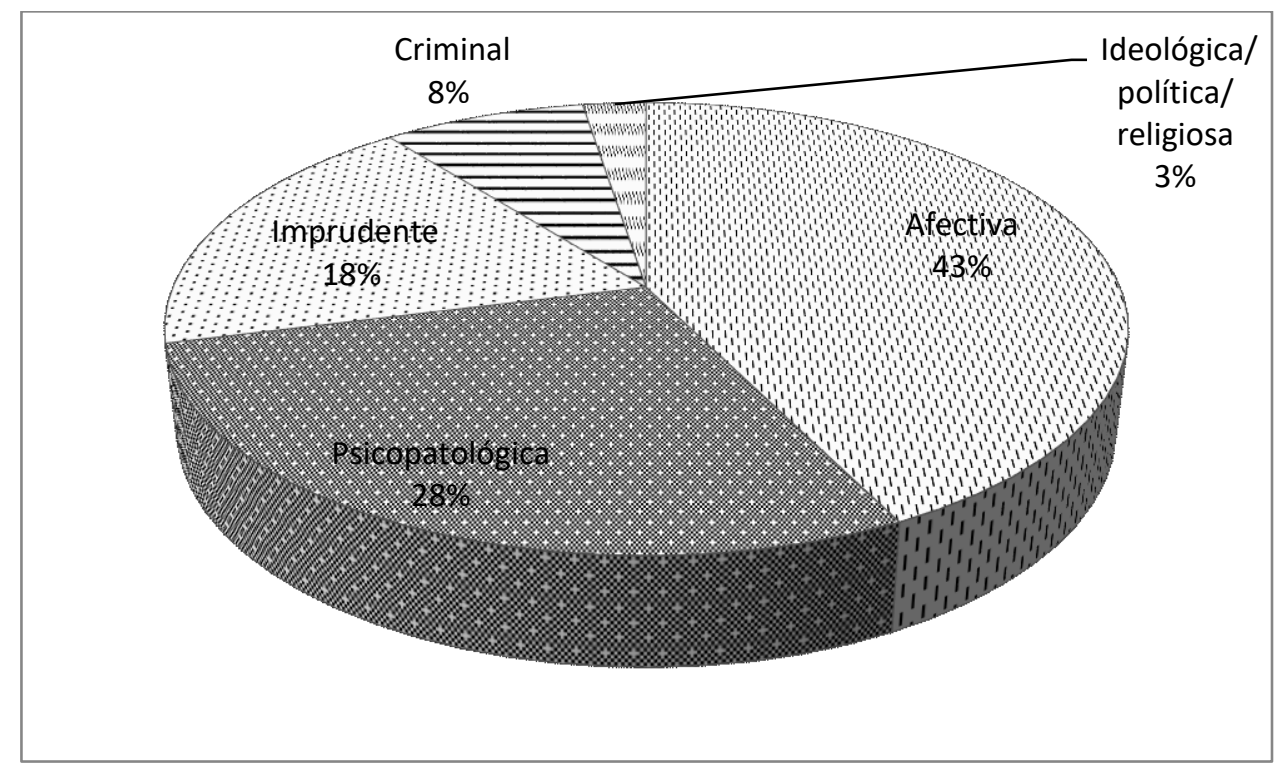


Tabla $N^{\circ} 5$. Modelo de regresión logística con variable dependiente Opinión compatible con imputabilidad comprometida

\begin{tabular}{|l|r|r|r|r|r|r|r|r|}
\hline $\begin{array}{l}\text { Variables } \\
\text { independientes }\end{array}$ & \multicolumn{1}{|c|}{$B$} & \multicolumn{1}{c|}{$D E$} & Wald & gl & \multicolumn{1}{|c|}{$p$} & OR & \multicolumn{2}{|l|}{$\begin{array}{c}\text { I.C. 95\% para } \\
\text { EXP(B) }\end{array}$} \\
\cline { 3 - 9 } & & & & & & & $\begin{array}{r}\text { Infer } \\
\text { ior }\end{array}$ & $\begin{array}{c}\text { Superi } \\
\text { or }\end{array}$ \\
\hline Conducta Bizarra & 4,2 & 1,2 & 12,9 & 1 & $<0,001$ & 67,5 & 6,8 & 669,9 \\
\hline $\begin{array}{l}\text { Motivación } \\
\text { Patológica }\end{array}$ & 4 & 0,8 & 24,7 & 1 & $<0,001$ & 57,2 & 11,6 & 281,7 \\
\hline Motivación Afectiva & 2,2 & 0,6 & 13,9 & 1 & $<0,001$ & 8,6 & 2,8 & 26,6 \\
\hline Autolesiones & 2,9 & 1,1 & 6,5 & 1 & 0,011 & 18,5 & 2 & 176,8 \\
\hline Daños & $-2,3$ & 1,1 & 4,1 & 1 & 0,042 & 0,1 & 0,01 & 0,9 \\
\hline Colaboración & 1,7 & 0,5 & 10,9 & 1 & 0,001 & 5,3 & 2 & 14,5 \\
\hline
\end{tabular}

Nota. DE: desvío estándar. gl: grados de libertad. OR: odds ratio 


\section{Referencias bibliográficas.}

'Hollweg M. Arson. ¿A disorder impulse control? Gesundheitswesen. 1994; 56(6):330-4.

"Rechlin T, Weis M. Empirical findings with arson offenders. Nervenarzt. 1992; 63(11):683-90.

iii Bradford JM. Arson: a clinical study. Can J Psychiatry.1982; 27(3):188-193.

iv Yesavage JA, Benezech $\mathrm{M}$, Ceccaldi $\mathrm{P}$ et al. Arson in mentally ill and criminal populations. J Clin Psychiatry. 1983; 44:128-30.

vLaubichler W, Kûhberger A. The role of alcohol in pyromania and arson.

Blutalkohol. 1995; 32 (4):208-17.

vi Laubichler W, Kûhberger A, Sedlmeier P. Pyromania and arson: A psychiatric and criminologic data analyses. Nervenarzt. 1996; 67(9):774-80.

vii Räsänen $P$, Hakko $A$, Vaisanen $E$. Arson trend increasing: a real challenge to psychiatry. J Forensic Sci. 1995a; 40(6):976-9.

viii Räsänen P, Hirvenoja R, Hakko H, Väisänen E. A portrait of the juvenile arsonist. Forensic Sci Int.1995b; 73(1):41-7.

ix Noreik K, Grûnfeld B. Forensic psychiatric examination of arsonists. Tidsskr Nor Laegeforen.1990; 110(14):1820-2.

${ }^{\times}$Rix KJ. A psychiatric study of adult arsonist. Med Sci Law. 1994; 34(1):21-34.

xi Smith J, Short J. Mentally disordered fire setters. Br J Hosp Med. 1995; 53(4):136-40.

xii Koppmann A, Gabler G, Portilla, D Aravena, O. Conducta incendiaria en Chile: Aspectos Clínicos y Conceptuales. Revista Española de Psiquiatría Forense, Psicología Forense y Criminología (Revista electrónica), 1998 (consultado 04-04-2013): Disponible en:

http://webcache.googleusercontent.com/searchCONDUCTAINCENDIARIA ENCHILE: ASPECTOS CLINICOS Y CONCEPTUALES. www.google.cl.

xiii Ritchie EC, Huff TG. Psychiatric aspects of arsonists. J Forensic Sci. 1999; 44: 733-40.

xiv Ulrich S, Borkenau P, Marneros A. Personality disorders in offenders: categorical versus dimensional approaches. J Pers Disord. 2001; 15(5):442-9.

${ }^{x v}$ Lindberg N, Holi MM, Tani P, Virkkunen M. Looking for pyromania; characteristics of a consecutive sample of Finnish male criminals with histories 
of recidivists firesetting between 1973 and 1993. BMC Psychiatry. 2005; 14: 547.

xvi Plinsinga AE, Colon EJ, de Jong S. Arson is usually not pyromania. Ned Tijdschr Geneeskd. 1997; 141(3):129-31.

xvii Shavindra RD, Jayn M. Arson and psychiatry, Sri Lanka Journal of Forensic Medicine, Science and Law. 2010; Vol 1 No 1.

xvii Virkunnen M. Reactive hypoglycemic tendency among arsonist. Acta Psychiatr Scand. 1984; 69(5):445-52.

xix Dolan M. Personality and neuropsychological function in violent, sexual and arson offenders. Med Sci Law.2002; 42(1):34-43.

xx Bourget D, Bradford JM. Female arsonist: a clinical study. Bull Am Acad Psychiatry Law. 1989; 17(3):293-300.

xxi Swinton M, Ahmed A. Arsonist in maximum security: mental state at the time of firesetting and relationship between mental state and pattern of behaviour, Med Sci Law 2001; 41(1):51-7.

xxi Ganon TA. Female arsonists: key features, psychopathology and treatment needs. Psychiatry. 2010; 73(2):173-89.

xxiii Räsänen $P$, Hakko A, Vaisanen $E$. The mental state of arsonists as determined by forensic psychiatric examination. Bull Am Acad Psychiatry Law. 1995c; 23(4):547-53.

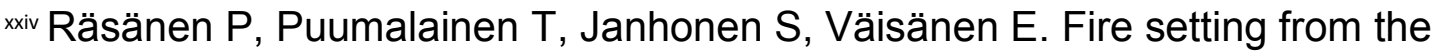
view point of an arsonist. J Psychosoc Nurs Ment Health Serv.1996; 34(3):1621.

${ }^{x}$ Hill RW, Langevin R, Paitich D, Handy L, Russon A, Wilkinson L. Is arson an aggressive act or a property offence? A controlled study of psychiatry referrals. Can J Psychiatry. 1982; 27(8):648-54.

xxvi Stewart LA. Female firesetters: Implications for treatment. Br J Psychiatry. 1993; 163:248-56.

xxvii Noblet S, Nelson B. A psychosocial approach to arson - a case control study of female offenders", Med Sci Law. 2001; 41(4):325-30.

xxviii Soothill, K. The criminal careers of arsonists. Med Sci Law. 2004; 44(1):2740.

xxix Enayati J, Grann M, Lubbe S, Faze S. Psychiatric morbidity in arsonists referred for forensic psychiatric assessment in Sweden. The Journal of Forensic Psychiatry and Psychology. 2008; 19 (2): 139-147. 
xx Harmon RB, Rosner R et Wiederligth M. Women and arson: a demographic study. J Forensic Sci. 1985; 30: 467-77.

xaxi Wilson RC. Why do women commit arson? CMAJ. 1988; 139(2):103-4.

xxxii Prentky RA, Knight RA, Sims-Knight JE, Straus H, Rokous F, Cerce D. Developmental antecedents of sexual aggression. Developmental Psychopathology. 1989; 1: 153-169.

xxiii Ward T, Hudson S, Marshall W. Attachment style in sex offenders: a preliminary study. The Journal of Sex Research. 1996; 1 (1), 17-26.

xxiv Ward T, McCormack J, Hudson S. Sexual offenders' perceptions of their intimate relationships. Sexual Abuse: A Journal of Research and Treatment. 1997; 9 (1), 57- 74.

xxx Lyn T, Burton L. Attachment, anger and anxiety of male sexual offenders. Journal of Sexual Aggression. 2005; 1:127-137.

xxxvi Código Penal, República de Chile. Editorial Jurídica de Chile. Vigésima edición; 2006.

xxxvii Servicio Médico Legal de Chile, Unidad de Archivo y Estadísticas. Anuarios. $1999-2012$.

xxyvii Hare RD. The Hare Psychopathy Checklist-Revised. Second Edition. Toronto: Multi-Health Systems. 2003.

xxix Folino JO. Trastorno antisocial de la personalidad en prisioneros. Rev Fac Cs Med Universidad Nacional de La Plata (revista electrónica), 2003 (consultado en 30.06.2016): Disponible en: http//revistamedicina.port5.com/articulos/100404.pdf.

${ }^{x l}$ Folino JO, Hare RD. Listado revisado para verificación de la psicopatía: su estandarización y validación en la Argentina. Acta Psiquiatr Psicol Am Lat. 2005; 51:94-104.

xi León-Mayer E, Cortés Olavarría MS, Folino JO. Descripción multidimensional de población carcelaria chilena. Psicoperspectivas. 2014;13: 68-81.

xlii Folino JO. Trastornos mentales y criminalidad: estudio de incidencia y características. Cátedra de Psiquiatría, Facultad de Ciencias Médicas, Universidad Nacional de La Plata; 1989.

xliii Dresdner R; A Aliaga; O Gutiérrez, M Martínez. Perfil de Agresores Sexuales. Revista de Psiquiatría y Ley. 2005; 2 (2): 76-84.

xiv Folino JO, Apezteguía M. Los homicidas. Rev Mex Prevención y Readapt Soc. 2000; 7:17-48 